\title{
Revisiting the consequences of inadequate Canadian physician resource planning: a renewed call to action
}

\author{
Robert Byrick, MD (D) Douglas Craig, MD • Franco Carli, MD, MPhil
}

Received: 21 June 2016/Accepted: 4 August 2016/Published online: 10 August 2016

(C) Canadian Anesthesiologists' Society 2016

The title for this editorial was purposely chosen to replicate the title for a 1999 editorial $^{1}$ published in the Journal by two of the current authors. Our goal in doing so is to emphasize that the deficiencies in anesthesia physician resource planning (PRP) identified almost twenty years ago remain largely unresolved. In their recent editorial in the Journal, Siu et $a .^{2}$ suggest that there is current uncertainty in the state of supply and demand for anesthesiologists.

Although Siu et al. recognize the importance of planning, they did not outline the principles that must be considered in any manpower planning model. These principles, some of which actually represent deficiencies in common approaches, must be recognized if we are to undertake PRP for the specialty of anesthesia. Deficiencies identified in PRP models in 1999 included the simplistic use of "head counts" rather than full-time equivalents (FTEs) of providers for the assessment of physician "stocks"; failure to consider changing demands in patient care (e.g., population growth, effects of aging on the need for surgical and anesthesia services); failure to consider

\footnotetext{
R. Byrick, MD

Department of Anesthesia, University of Toronto, Toronto, ON, Canada

R. Byrick, MD ( $\square)$

Department of Anesthesia, St. Michael's Hospital, 30 Bond St., 1DN, Toronto, ON M5B 1W8, Canada

e-mail: Robert.byrick@utoronto.ca

D. Craig, MD

Department of Anesthesia, University of Manitoba, Winnipeg, MB, Canada

F. Carli, MD, MPhil

Department of Anesthesia, McGill University Health Centre, Montreal, QC, Canada
}

changing roles of anesthesiologists within the Canadian healthcare system; and the projected flows into and out of the pool of active anesthesiologists. Indeed, our original 1999 editorial $^{1}$ called for a collaborative approach to Canadian anesthesia PRP that would allow the profession to participate actively in discussions that would ideally be evidence-based. Such a comprehensive collaborative approach has yet to happen, and we argue that the current situation discussed by Siu et al. ${ }^{2}$ reflects this lack of a proactive approach by our specialty.

Following a discussion led by the Association of Canadian University Departments of Anesthesia (ACUDA) regarding the 1999 editorial, Ms Eva Ryten, Former Director of Research (now retired), Association of Canadian Medical Colleges, developed a planning model. ${ }^{\mathrm{A}}$ Ms Ryten's report and the linked summaries of both the anesthesia service needs ${ }^{3}$ and the anesthesia workforce ${ }^{4}$ were subsequently used in discussions to encourage changes that would address the shortage of anesthesiologists in 2002. Participants in these discussions included provincial Ministries of Health, the Royal College of Physicians and Surgeons of Canada (RCPSC), provincial licensing bodies, the Canadian Medical Association (CMA), and the Canadian Anesthesiologists' Society (CAS). These discussions resulted in significant increases in residency positions in many centres and a change in the model of anesthesia practice to include anesthesia assistants.

The primary focus of the Ryten study was an analysis of the province of Quebec's billing data for all anesthesia

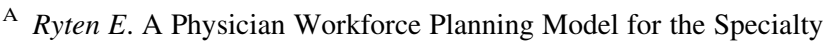
of Anesthesia: Theoretical and Practical Considerations. Association of Canadian University Departments of Anesthesia. Available from URL: http://www.cas.ca/English/Page/Files/93_Ryten\%20Report.pdf (accessed June 2016).
} 
services during one full year (fiscal 1998-99). These data were made available to ACUDA by the Régie de l'assurance maladie du Québec (RAMQ). Separation of billings by patient age and sex allowed identification of the cost per capita in each grouping. Ontario Health Insurance Plan (OHIP) anesthesia billing data for 1998-99 were found to be consistent with the Quebec data, ${ }^{3}$ validating the approach. Statistics Canada population data for 1999 as well as projections through to 2016 were used to extrapolate the Quebec data to all provinces, allowing estimates of then current (1999) and future (through to 2016) service needs. The projected future service requirements were based on both population numbers and the predicted demographic changes (i.e., sex and age). Demand for anesthesia services was anticipated to increase by an estimated $30.9 \%$, with $17.9 \%$ linked to population increases and the remainder due to an aging population. The billing data were also used to estimate the number of FTE anesthesiologists that would be required to provide the services at the various time points.

The CMA physician database at the time provided anesthesiologist numbers $(2,287)$ and ages as of January 1 , 2000. Ryten's needs assessment (for both clinical and nonclinical activities) identified a requirement for 2,495 FTE anesthesiologists in 1999 and 3,265 FTE in 2016. ${ }^{3}$ Taking into account the ages of the anesthesiologists in 2000, the projected future service requirements, and the expected additions to the pool of anesthesiologists following completion of the specialty training program (at the then-current numbers), a deficit of more than 650 FTEs was projected for the period 2000-2016.

Ryten went to great lengths in her report to explain the basic theories and limitations of physician human resource planning. She cautioned that, "if a physician workforce study is carried out, but no testing of the model takes place thereafter or no retrospective checking or updating takes place, no matter how good the initial study, it is not a planning model. It is perhaps the first stage of the complete process." Unfortunately, the mandatory testing, validation, and revision of the model described in the Ryten report has not happened. As a result, in 2016, many anesthesiologists think that there are too many providers being trained, and we continue to lack a comprehensive mechanism to analyze anesthesia PRP issues for Canada.

The editorial by Siu et al. ${ }^{2}$ serves as a reminder of this issue and it is also timely, since Ryten chose 2016 as the year for her long-term projections of anesthesia provider requirements and planning. We recognize that Siu et $a .^{2}$ did not attempt to review the state of PRP planning but instead presented the pros and cons of anesthesiologist surpluses and shortages. Unfortunately, the authors seem to take a passive approach and do not emphasize the essential need for a coordinated proactive and prospective approach to collect data and drive decision-making. The proven usefulness of such data in discussions with influential organizations outside the profession seems to have been forgotten. Although Siu et al. ${ }^{2}$ suggest that "economic concerns may be the major contributor to the supply / demand imbalance" perceived today, they base this on the restricted growth of hospital budgets, leading to reduced hospital resources and hence fewer positions available.

We certainly acknowledge that perceived job shortages are a very real concern for recent graduates of anesthesia residency programs, but it is important to stress that data are lacking to suggest that there is a diminishing patient need for anesthesia care services. The issue of an imbalance in physician manpower is not restricted to only anesthesiology. As the RCPSC reported in 2013, there has been an excess of graduates from many surgical specialty training programs when compared with the permanent positions available for them in the Canadian health system. ${ }^{\mathrm{B}}$ This is true even for specialties where there are substantial patient waiting lists for surgery, indicating that considerable need still exists in the current population.

Ryten's model clearly showed that the predominant causes of future increases in demand for anesthesia services were population growth and demographic shifts (i.e., aging). If this fundamental lesson remains true today, and in our view it still does, we need current data to determine the appropriate actions to drive PRP for the specialty. In the long term, the needs of the population for anesthesia (and surgical) services will determine demand, and a relatively short-term and geographically specific shortage of jobs is not an accurate surrogate marker for an adequate number of providers. The reported job shortage may reflect only a transient maldistribution of providers within Canada or the inadequate capacity of the healthcare system to meet current patient needs. Patients will ultimately insist on access to adequate service. If the current healthcare system cannot provide the service, then alternate methods and sites, such as out-of-hospital premises (OHP), will be developed and regulated to provide the service, and anesthesiologists will need to be trained to work safely in this environment. One consequence is the need for a detailed discussion in our postgraduate programs as regards the challenges of OHP anesthetic practice. The growth of OHP regulation in various provinces (e.g., British Columbia, Alberta and, more recently, Ontario, Manitoba, and others), illustrates that this trend is important. ${ }^{5}$

\footnotetext{
B Royal College of Physicians and Surgeons of Canada. What's really behind Canada's unemployed specialists? Too many, too few doctors? Findings from the Royal College's employment study2013. Available from URL: http://www.royalcollege.ca/rcsite/ documents/health-policy/employment-report-executive-summary-2013e.pdf (accessed June 2016).
} 
In 2005, Donen and Hardy ${ }^{6}$ thoughtfully reflected on the PRP issue in anesthesia and challenged Canadian anesthesia organizations to "spearhead" a national process to develop a standardized approach to PRP needs. The fact that Siu et $a l^{2}{ }^{2}$ report the problem in 2016 strongly suggests that our specialty organizations did not accept their challenge to follow up on the initial experience of using Ryten's data to drive change. We are not implying that the Ryten study should be ongoing or even repeated. It was costly and complex and required dedicated full-time expertise. We agree with Donen and Hardy $^{6}$ that Canadian anesthesia organizations should demonstrate leadership in developing a national process to maintain a standardized approach to anesthesia PRP for the purposes of documenting objective evidence for anesthesia requirements over time. It is somewhat ironic that we live in the most data-rich era in the history of our specialty, and yet, we do not take full advantage of data to plan the future of our own profession. Failing to do so will result in perpetuating the cycle, with either the Ministry of Education or Health in each province making uninformed unilateral decisions. Their decisions could impact the number of available anesthesia providers for decades and potentially influence the manner in which anesthesia is provided to patients. If the profession does not undertake the development of a comprehensive PRP model and contribute evidence that will impact PRP decisions, we will then have no means to lament the results of such decisions or to argue forcefully against them in the future.

\section{Les conséquences d'une mauvaise planification des ressources médicales canadiennes: un appel renouvelé à agir}

Le titre de cet éditorial a été volontairement choisi pour reprendre celui d'un éditorial ${ }^{1}$ de 1999 publié dans le Journal par deux des auteurs actuels. En faisant cela, notre but est d'insister sur le fait que les lacunes dans la planification des ressources en médecins (PRM) en anesthésie, identifiées il y a près de vingt ans, ne sont toujours pas résolues. Dans un récent éditorial publié par le Journal, Siu et coll. ${ }^{2}$ suggèrent qu'il existe présentement une incertitude sur l'état de l'offre et de la demande pour les anesthésiologistes.

Même si Siu et coll. reconnaissent l'importance de la planification, ils n'ont pas évoqué les principes qui doivent être pris en compte dans tout modèle de planification en ressources humaines. Ces principes, dont certains constituent une lacune dans les approches courantes, doivent être reconnus si nous voulons entreprendre une PRM pour la spécialité de l'anesthésie. Les erreurs identifiées dans les modèles de PRM de 1999 incluaient notamment une utilisation simpliste du « nombre de têtes » plutôt que des équivalents temps-pleins (ÉTP) pour l'évaluation des «stocks » de médecins, la non prise en compte des exigences changeantes dans les soins aux patients (par exemple, l'accroissement de la population, les conséquences du vieillissement sur les besoins de services chirurgicaux et anesthésiques), la non prise en compte des changements intervenus dans le rôle des anesthésiologistes au sein du système de soins de santé canadien et, enfin, la projection des entrées et sorties de médecins du « réservoir » d'anesthésiologistes actifs. De fait, notre éditorial initial de $1999^{1}$ lançait un appel à une approche collaborative de la PRM en anesthésie au Canada, qui permettrait à la profession de participer activement à des discussions qui s'appuieraient, idéalement, sur des données probantes. Une telle approche collaborative complète tarde à se produire, et nous croyons que la situation actuelle décrite par Siu et coll. ${ }^{2}$ reflète cette absence de démarche proactive par notre spécialité.

Après une discussion menée par l'Association des départements universitaires canadiens en anesthésie (ACUDA) sur l'éditorial de 1999, Mme Eva Ryten, ancienne directrice de la recherche à l'Association des collèges médicaux canadiens (aujourd'hui retraitée), a élaboré un modèle de planification. ${ }^{\mathrm{A}}$ Le rapport de Mme Ryten, ainsi que les résumés des besoins en services d'anesthésie ${ }^{3}$ et des effectifs en anesthésie, ${ }^{4}$ ont ensuite fait l'objet de discussions pour encourager des changements qui répondraient au manque d'anesthésiologistes en 2002. Les participants à ces discussions étaient notamment les ministères provinciaux de la Santé, le Collège royal des médecins et chirurgiens du Canada (CRMCC), les organismes provinciaux de gestion des licences, l'Association médicale canadienne (AMC) et la Société canadienne des anesthésiologistes (SCA). Ces discussions ont abouti à des augmentations significatives du nombre de postes de résidence dans de nombreux centres et à un changement du modèle de la pratique de l'anesthésie pour inclure des assistants en anesthésie.

L'étude de Mme Ryten s'était principalement centrée sur une analyse des données de facturation de la province du Québec pour tous les services d'anesthésie au cours d'une année complète (année fiscale 1998-99). Ces données avaient été mises à la disposition de l'ACUDA par la Régie de l'assurance maladie du Québec (RAMQ). Une analyse détaillée de la facturation par âge et sexe des patients a permis d'identifier un coût par personne dans chaque groupe. Les données de facturation d'anesthésie du 
régime d'assurance maladie de l'Ontario (OHIP_Ontario Health Insurance Plan) pour 1998-1999 se sont avérées similaires aux données québécoises, ${ }^{3}$ validant ainsi cette démarche. Les données de population de Statistiques Canada pour 1999 ainsi que des projections jusqu'à 2016 ont été utilisées pour extrapoler les données du Québec à toutes les provinces, permettant ainsi d'estimer les besoins de services actuels (1999) et futurs (jusqu'à 2016). Les besoins futurs projetés étaient basés à la fois sur le nombre d'habitants et sur les prédictions de variations démographiques (c'est-à-dire, sexe et âge). Il était anticipé que la demande de services d'anesthésie augmenterait d'environ $30,9 \%$, dont $17,9 \%$ liés à l'augmentation de la population, et le reste en rapport avec son vieillissement. Les données de facturation ont également servi à estimer le nombre d'anesthésiologistes ÉTP qui seraient nécessaires pour fournir les services à différents moments dans le temps.

La base de données des médecins de l'AMC avait alors fourni le nombre (2 287) et l'âge des anesthésiologistes au $1^{\mathrm{er}}$ janvier 2000. L'évaluation des besoins par Mme Ryten (pour, à la fois, les activités cliniques et non cliniques) a identifié un besoin de 2495 anesthésiologistes ÉTP en 1999 et 3265 ÉTP en 2016. ${ }^{3}$ En prenant compte de l'âge des anesthésiologistes en 2000, de la projection des besoins en services futurs et de l'arrivée attendue d'anesthésiologistes terminant leur programme de formation spécialisée (selon les nombres alors existants), elle prévoyait un déficit de plus de 650 ÉTP pour la période 2000-2016.

Mme Ryten a poussé très loin son travail pour expliquer les bases théoriques et les limites de la planification des ressources humaines en médecins. Elle a mis en garde en ces termes: "Si une étude est menée sur les effectifs médicaux, mais qu'aucun test du modèle n'a lieu par la suite, ou si aucun contrôle rétrospectif ou aucune mise à jour n'a lieu, peu importe la qualité du travail initial, il ne s'agit plus d'un modèle de planification. C'est peut-être la première étape d'un processus complet. » Malheureusement, la vérification, la validation et la révision nécessaires du modèle décrit dans le rapport Ryten n'ont pas eu lieu. Par conséquent, en 2016, de nombreux anesthésiologistes sont d'avis que l'on forme trop de professionnels de la santé, et nous n'avons toujours pas de mécanisme global permettant d'analyser les questions de PRM en anesthésie au Canada.

L'éditorial de Siu et coll. ${ }^{2}$ sert de rappel sur ce problème et il vient à point nommé, puisque Mme Ryten avait choisi 2016 comme année pour ses projections à long terme des besoins et de la planification des ressources en soins d'anesthésie. Nous reconnaissons que Siu et coll. ${ }^{2}$ n'ont pas tenté de revoir l'état de la PRM, mais qu'ils ont plutôt présenté les avantages et les inconvénients des surplus et manques d'anesthésiologistes. Les auteurs semblent avoir regrettablement adopté une démarche passive et n'insistent pas sur le besoin essentiel d'une approche proactive et prospective coordonnée, pour collecter des données et orienter la prise de décision. L'utilité prouvée de telles données dans les discussions avec des organisations influentes en dehors de la profession semble avoir été oubliée. Bien que Siu et coll. ${ }^{2}$ suggèrent que «les préoccupations économiques puissent contribuer de façon majeure au déséquilibre offre/demande » perçu aujourd'hui, ils basent cette opinion sur les restrictions de croissance des budgets des hôpitaux qui aboutissent à une baisse de leurs ressources et, par conséquent, à une diminution des postes disponibles.

Nous reconnaissons certes que le manque perçu de postes disponibles est une préoccupation bien réelle pour les récents diplômés des programmes de résidence en anesthésie, mais il est important de souligner que nous manquons de données pour suggérer qu'il existe une baisse des besoins des patients pour des services de soins anesthésiques. Le problème des déséquilibres dans les effectifs médicaux n'est pas limité à la seule anesthésiologie. Comme l'a signalé le CRMCC en 2013, il $\mathrm{y}$ a eu un excès de diplômés dans de nombreux programmes de formation en spécialités chirurgicales par rapport au nombre de postes qui leur étaient ouverts dans le système de santé canadien. ${ }^{\mathrm{B}}$ Cela est même valable pour des spécialités où existent des listes d'attente substantielles pour la chirurgie, indiquant qu'il existe encore un besoin considérable dans la population actuelle.

Le modèle de Mme Ryten a clairement démontré que les causes prédominantes des augmentations futures de la demande pour des services d'anesthésie étaient l'augmentation de la population et les changements des caractéristiques démographiques (c'est-à-dire, le vieillissement de la population). Si cette leçon fondamentale reste vraie aujourd'hui-et à notre avis, c'est toujours le cas-, nous avons besoin de données actuelles pour définir les actions qu'il convient de prendre pour orienter la PRM dans notre spécialité. À long terme, les besoins de la population en services anesthésiques (et chirurgicaux) détermineront la demande et un manque de postes à relativement court terme et dans une région géographiquement spécifique, n'est pas un indicateur fiable d'un nombre adéquat d'anesthésiologistes. Le manque de postes signalé pourrait ne refléter qu'une mauvaise répartition transitoire des professionnels dans l'ensemble du Canada, ou l'incapacité du système de soins de santé à répondre aux besoins actuels des patients. Les patients finiront par insister pour avoir accès à un service adéquat. Si le système actuel de soins de santé ne peut pas fournir le service, alors des méthodes et sites alternatifs, tels que des établissements extra-hospitaliers (OHP-out-of-hospital 
premises) seront développés et réglementés pour fournir les services, et les anesthésiologistes devront être formés pour travailler de façon sécuritaire dans un tel environnement. Une conséquence de cette situation est le besoin d'une discussion détaillée dans nos programmes de formation supérieure, concernant les défis de la pratique anesthésique en OHP. La réglementation de plus en plus importante concernant les OHP dans différentes provinces (ColombieBritannique, Alberta et, plus récemment, Ontario, Manitoba et d'autres) démontre que cette tendance est importante. ${ }^{5}$

En 2005, Donen et $\mathrm{Hardy}^{6}$ ont mené une réflexion approfondie sur le problème de la PRM en anesthésie et ont mis au défi les organisations canadiennes en anesthésie de devenir le fer de lance d'un processus national d'élaboration d'une démarche standardisée pour l'évaluation des besoins de PRM. Le fait que Siu et coll. ${ }^{2}$ relèvent le problème en 2016 laisse fortement entendre que nos organisations professionnelles n'ont pas relevé le défi qui leur était lancé de poursuivre l'expérience initiale d'utiliser les données de Mme Ryten pour orienter le changement. Nous ne suggérons pas de poursuivre ou même de répéter l'étude Ryten. Elle fut complexe et coûteuse, et on a dû lui consacrer une expertise à plein temps. Nous sommes d'accord avec Donen et $\mathrm{Hardy}^{6}$ pour dire que les organisations canadiennes d'anesthésie devraient faire preuve de leadership en élaborant un processus national destiné à maintenir une démarche standardisée de la PRM en anesthésie, afin d'accumuler des données objectives sur les besoins de la spécialité au fil des années. Il est quelque peu ironique que nous vivions dans l'ère la plus riche en données de toute sorte dans toute l'histoire de notre spécialité, et que nous ne puissions néanmoins pas profiter de ces données pour planifier le futur de notre propre profession. Ne pas le faire nous amènera à perpétuer un cycle, où le Ministère de l'Éducation ou celui de la santé de chaque province prendront des décisions unilatérales, sans informations. Leurs décisions pourraient avoir un impact sur le nombre d'anesthésiologistes pour des décennies et potentiellement influencer la façon dont l'anesthésie est prodiguée aux patients. Si la profession n'entreprend pas l'élaboration d'un modèle complet de PRM et ne contribue pas aux données qui auront un impact sur les décisions de PRM, nous n'aurons à l'avenir aucun moyen de nous lamenter sur les résultats de telles décisions ou de les contester avec force.

Acknowledgements The authors express our thanks to Drs C. David Mazer and Matt Kurrek for their helpful comments on the manuscript.

\section{Conflicts of interest None declared.}

Editorial responsibility This submission was handled by Dr. Hilary P. Grocott, Editor-in-Chief, Canadian Journal of Anesthesia.

Remerciements Les auteurs tiennent à remercier le Dr C. David Mazer et le Dr Matt Kurrek pour leurs utiles commentaires sur ce manuscrit.

Conflits d'intérêts Aucun déclaré.

Responsabilité éditoriale Cette soumission a été traitée par le Dr Hilary P. Grocott, rédacteur en chef, Journal canadien d'anesthésie.

\section{References}

1. Byrick RJ, Craig DB. Consequences of inadequate Canadian physician resource planning. Can J Anesth 1999; 46: 913-8.

2. Siu E, Tenenbein P, Kavanagh BP. Job shortages in anesthesia: a pro or con? Can J Anesth 2016; 63: 529-36.

3. Byrick RJ, Craig D, Carli F. A physician workforce planning model applied to Canadian anesthesiology: assessment of needs. Can J Anesth 2002; 49: 663-70.

4. Craig D, Byrick R, Carli F. A physician workforce planning model applied to Canadian anesthesiology: planning the future supply of anesthesiologists. Can J Anesth 2002; 49: 671-7.

5. Kurrek MM, Twersky RS. Office-based anesthesia. Can J Anesth 2010; 57: 256-72.

6. Donen N, Hardy JF. Anesthesia physician resources-time to change the focus. Can J Anesth 2005; 52: 1-7. 